\title{
Der derzeitige weibliche
}

Zuchttypus des schweizerischen

Braunviehes, dargestellt

mittels der Körpermasse und

der Lebendgewichte von

ausgesprochenen Rassetieren

Doctoral Thesis

Author(s):

Sciuchetti, Andrea

Publication date:

1933

Permanent link:

https://doi.org/10.3929/ethz-a-000099229

Rights / license:

In Copyright - Non-Commercial Use Permitted 
Diss. ETH: $75 \wedge \quad B$

\section{Der derzeitige weibliche Zuchttypus} des schweizerischen Braunviehes, dargestellt mittels der Körpermaße und der Lebendgewichte
von ausgesprochenen Rassetieren.

Von der Eidgenössischen Technischen Hochschule in Zürich zur Erlangung der

Würde eines Doktors der technischen Wissenschaften genehmigte

Nr. $751 \quad$ Promotionsarbeit

vorgelegt von

Andrea Sciuchetti

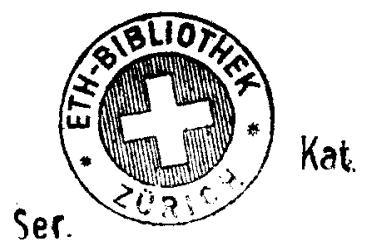
aus Braggio (Graub.).

Referent: Herr Prof. Dr. A. Schmid.

Korreferent: Herr Prof. Dr. M. Küpfer.

Verlag Paul Parey 
Auf Grund dieser Gegenüberstellung läßt sich folgendes sagen:

Zufolge des absolut kurzen Rumpfes nähert sich das Format des Vorarlbergerrindes mehr der Quadratform als das des Schweizer Braunviehes.

Der größere Brustquerschnitt des Zuchttypus der schweizerischen Braunviehrasse weist auf eine bessere Rippenwölbung bei dieser Rasse hin.

Der kleinere seitliche Rumpfformat- und Rumpfgrundflächenindex bei meiner Population rührt vom außerordentlich kurzen Rumpfe des Allgäuerrindes her.

Die auf die Widerristhöhe reduzierte Brusttiefe des Vorarlbergerrindes ist jener der Schweizer Ausgangsrasse um ein geringes überlegen.

Das Becken ist beim schweizerischen Braunvieh besser entwickelt als beim Allgäuervieh.

Die Kopflänge ist, relativ betrachtet, bei beiden Rassen ungefähr gleich.

Der Metakarpalindex weist auf eine gleiche relative Knochenstärke hin.

Betrachten wir nun den Typus der Vorarlberger und der Schweizer Braunviehrasse als Ganzes, so geht aus dem Gesagten hervor, daß das Vorarlbergerrind relativ kurz, flach und hochbeinig ist, dagegen relativ gute Brusttiefenmaße besitzt. Das frühreifere Schweizer Braunvieh seinerseits weist besonders eine absolut und relativ bessere Breiten- und Längenentwicklung auf. Diese Befunde lassen auf eine höhere Züchtungsstufe des Schweizerrindes schließen. Der Typus des Vorarlbergerindes hingegen trägt die charakteristischen Kennzeichen einer primitiveren $Z$ üchtungsstufe an sich. Inwiefern nun dieser Typus die Folge einer bewuBten Selektion oder primitiverer Haltung, insbesondere extensiverer Ernährung ist, kann hier nicht näher verfolgt werden.

\section{Zusammenfassung und SchluBfolgerungen.}

Die vorliegende Untersuchung stellte sich folgende Aufgaben:

die zahlenmäßige Darstellung der morphologischen Ausgestaltung des weiblichen Zuchttypus der schweizerischen Braunviehrasse mittels der Körpermaße und des Lebendgewichtes einer genügend großen Zahl von ausgesprochenen Rassevertretern,

die Feststellung der Körperproportionen ausgewachsener Zuchtkühe genannter Rasse,

die Ermittlung des Durchzüchtungsgrades der Rasse und die Umschreibung der für jedes Maß zulässigen Schwankungsbreite (Standard im weiteren
Sinne),

die Charakterisierung allfälliger morphologischer Typusunterschiede zwischen ausgewiesenen Milchleistungskühen und Zuchtkühen ohne Milchleistungsausweis,

die Abklärung der Berechtigung, innerhalb der schweizerischen Braunviehzucht Untertypen zu unterscheiden,

wenn möglich Feststellung von im Laufe der letzten Jahrzehnte eingetretener Typusänderungen.

Unter dem Typus wird hier die Summe der sicht-, meß- und wägbaren morphologischen und physiologischen Merkmale eines Individuums, bezw. der
Rasse verstanden.

In Übereinstimmung mit A. Schmid (91) definierte ich den Zuchttypus exterieuristisch "als die bei der Verfolgung eines Zuchtzieles zutage tretende, äußere Erscheinungsform der Vertreter einer Rasse". Als Maßstab 
zur Überprüfung der Zuchttypuszugehörigkeit jedes einzelnen Prüflings wurde das Ergebnis der offiziellen Punktierbewertung (Minimalpunktzahl = Kantonsdurchschnitt + 3 Punkte) herangezogen.

Meine Untersuchung beschränkte sich also auf die Verfolgung der exterieuristis chen Merkmale der Prüflinge, welche immer noch die Basis zur Beurteilung des Zuchtwertes eines Tieres darstellen.

Die Messungen wurden an 389 ausgewachsenen Kühen (d. h. über 5 Jahre alt) ausgeführt.

Die allgemeinen und meßmethodischen Ergebnisse vorliegender Untersuchung lauten wie folgt:

Es erscheint zweckmäßig, die Messungen als wesentliches Hilfsmittel der Tierbeurteilung in Zukunft weiter auszubauen. Dadurch wird die Exterieurlehre auf eine objektivere Basis gestellt. Inskünftig sollte nicht nur auf Ausstellungen, sondern auch anläßlich von Schauen und in den Genossenschaften wieder mehr gemessen werden. Das Regulativ über die Durchführung der Leistungsprüfungen schweizerischer Rinderrassen durch die Viehzuchtverbände vom 1. Oktober 1932 bildet einen ersten vielversprechenden Anfang in dieser Richtung.

Die Tierkörpermessungen erfüllen ihren Zweck nur dann, wenn sie in die Hand von Fachleuten gelegt werden.

Die absoluten Maßangaben der offiziellen Meßkarte des Verbandes schweizerischer Braunviehzucht-Genossenschaften sind weiterhin beizubehalten. Dazu sollte die Meßkarte vorab durch folgende Maße ergänzt werden: Rückenhöhe, Hüfthöhe, Hüftgelenkhöhe, Gesäßhöckerhöhe, Höhe der Bugspitze, Ellenbogenhöhe, Höhe der Kniescheibe, Buggelenkweite, Gesäßweite, Thoraxlänge, Umfang des Metakarpus, Umfang des Metatarsus, Stirnlänge, Stirnbreite, Stirnenge, Ganaschenweite, Körpergewicht und Schulterwinkel.

Für wissenschaftliche Zwecke wäre zudem noch wünschbar, wenn folgende Maße genommen würden: Schulterlänge, Schulterbreite, Lendenlänge, Kreuzlänge, Oberarmbeinlänge, Unterarmbeinlänge, Länge des Metakarpus, Oberschenkellänge, Unterschenkellänge, Länge des Metatarsus, Sprunggelenkumfang, Horngrundumfang, Beckenwinkel nach hinten und Beckenwinkel nach der Seite.

Die Berücksichtigung einheitlicher Meßpunkte ist anzustreben, wobei man, soweit möglich, besonders bei praktischen Arbeiten, die offiziellen Ansatzstellen benützen sollte. Haben sich diese als falsch erwiesen, so sollte man auf Änderung dringen, und nicht den Wirrwarr durch Einführung neuer Meßpunkte vermehren.

Die Verarbeitung der erbrachten Einzelergebnisse hat zukünftig bei meßtechnischen Untersuchungen wissenschaftlichen Charakters stets mit Hilfe der biometrischen Methoden zu erfolgen. Diese allein vermitteln uns ein übersichtliches und erschöpfendes Bild der körperlichen Eigentümlichkeiten einer Population.

Als Vorbedingung für die Anwendung der Biometrik verlangt die Fehlertheorie mindestens eine Frequenz von 50 Individuen. Diese Zahl wechselt im übrigen je nach der Ausgeglichenheit des Untersuchungsmaterials.

Ein Gradmesser für die minimale, noch variationsstatistisch auswertbare Individuensumme bildet der kurvenmäßige, besser noch der rechnerische Vergleich der empirischen mit der idealen Verteilung mit entsprechendem Streungs- 
maß. Der Grad der Übereinstimmung hängt nicht allein von der Frequenz, sondern ebenso sehr von der Ausgeglichenheit der Population ab.

Stimmt eine Prüfungsreihe mit größerer Individuensumme mit der Gauss:schen Fehlerkurve nicht überein, so vermögen die erhaltenen Ergebnisse aus nicht immer feststellbaren Gründen die tatsächlichen Verhältnisse nicht eindeutig wiederzugeben.

Der Vergleich einer Prüfungsreihe mit einer anderen darf nicht allein auf Grund der Mittelwerte erfolgen, sondern es müssen gleichzeitig auch die mittleren Fehler der tatsächlich ermittelten Differenzen festgestellt werden. Beträgt die Differenz mehr als dreimal ihren mittleren Fehler, so ist dieselbe wesentlich. Das Nichtvorhandensein dieses Sicherheitsmaßes besagt, daß beide Populationen bezüglich genannter Eigenschaft zum gleichen Typus gehören.

Die Abgrenzung der für jedes Merkmal zulässigen Schwankungsbreite ist mittels der Standardabweichung möglich. Diese Methode ist meines Wissens die objektivste, die uns heute zur Verfügung steht, indem einzig die Feststellung ihres Parameters subjektiv bedingt ist.

Die bei einer Population im Raume des Mittelwertes zu erwartende Frequenz ist mit Hilfe einer Formel von P. Riebesell (87) feststellbar. Diese ist eine Funktion der Individuensumme und der Standardabweichung des betreffenden Merkmals.

Ein einziges Grundmaß als Vergleichsmaß ist fallen za lassen. Der Vergleich von zwei Populationen einer Rasse soll in erster Linie mit Hilfe der Mittelwerte bei Mitberücksichtigung ihrer Konstanten erfolgen. Die Gegenüberstellung der Maße von Einzeltieren mit dem Standard im weiteren Sinne hat mit Hilfe der absoluten Zahlen stattzufinden.

Die Berechnung von Indizes bildet eine notwendige Vervollständigung jeglicher meßtechnischen Untersuchung des lierkörpers. Diese geben von den uns heute bekannten Hilfsmitteln die Körperproportionen am vollständigsten wieder.

Für die Züchterschaft des schweizerischen Braunviehes mögen hauptsächlich folgende Feststellungen von Interesse sein:

Die von mir ermittelten Meßergebnis e erlauben eine zahlenmäßige Umschreibung des weiblichen Zuchttypus derschweizerischen Braunviehrasse.

Die Mittelwerte der 55 Maße und der 24 Indizes geben den Standard im enger en Sinne der Zuchtkuh der braunen Schweizerrasse wieder.

Die Mittelwerte weisen im allgemeinen auf eine für eine Höhenrasse e rwünschte Körperausbildung hin. Daneben werden auch andere Merkmale, wie z. B. die Schulterstellung des Schweizer Braunviehes umschriebeu, die als eher zu steil bezeichnet werden muß. Diese Tatsachen sollten bei der Beurteilung inskünftig noch mehr beachtet werden.

Die Form des Thoraxquerschnittes von einigen Kühen wird bildlich wiedergegeben. Die beiden Brusthälften sehen nicht symmetrisch aus, wobei besonders die rechte Seite eine stärkere Wölbung als die linke aufweist. Gleichzeitig wurde auch der Flächeninhalt des Thoraxquerschnittes bestimmt.

Die Neigung und die Abdachung des Beckens des weiblichen Zuchttypus der schweizerischen Braunviehrasse wird zahlenmäßig 
festgelegt. Die Beckenneigung beträgt $6,99 \pm \mathbf{0 , 1 7 0}$, während die Abdachung des Beckens mit der Horizontalen einen Winkel von $35,38 \pm 0,16^{\circ}$ bildet. Die trigonometrische Berechnung genannter Winkel ergab ungefähr um 2 Grad niedere Werte als die mit dem Goniometer ermittelten. Die direkte Winkelbestimmung ist der rechnerischen vorzuziehen.

Der Standard im engeren Sinne erlaubt die Feststellung vonzeitlich oder örtlich bedingten Typusänderungen und dient somit der Typkontrolle.

Die Variabilität der Maße, oder anders gesagt, der Grad der Ausgeglichenheit, wird durch die Variationskoeffizienten angegeben. Ich unterschied vier Variabilitäts stufen.

Die Höhenmaße weisen die geringste Variabilität auf, während die Hornmaße, das Lebendgewicht und der "Beckenwinkel nach hinten" die größte Unausgeglichenheit besitzen.

In Übereinstimmung mit den Ergebnissen von C. Kronacher, C. vo n Patow und W. Schäper an Harzer- und Ostfriesen Kühen zeigen die Maße meiner Prüfungsreihe in großen Zügen eine mehr oder weniger ausgeprägte Gesetzmäßigkeit im Bauplan des Rinderkörpers aller Rassen an.

Die Maximal- und Minimalwerte sämtlicher untersuchten Maße und berechneter Indizes sind bedeutend und erhellen die Notwendigkeit, die für typustreue Tiere zulässige Schwankungsbreite für die hauptsächlichsten Körperpartien und Indizes festzulegen.

Der Standard des weiblichen Vertreters der schweizerischen Braunviehrasse im weiteren Sinne wird zahlenmäBig umschrieben. Er beschlägt die Körpermaße und die Indizes. Das Zuchtziel erhält durch die Festsetzung dieser Schwankungsgrenzen einen Rahmen. Der Standard im weiteren Sinnedient zur Beurteilung, bezw. Typuskontrolle des Einzelindividu ums.

Die Übereinstimmung mit diesem Standard sollteals Vorbedingung für eventuelle Elitezuchtbucheintragungen und die Prämiierung in erster Klasse gefordert werden.

Die Aufstellung eines Standards im weiteren Sinne bezweckt, die Exterieurlehre mit wissenschaftlicher Hilfe in objektive Bahnen zu lenken.

Zwischen Milchleistungskühen und den Zuchtkühen ohne Milchleistungsausweis besteht ein mathematisch gesicherter Unterschied in der Rumpfund Brustlänge, und zwar in dem Sinne, daß die Milchleistungstiere im Rumpf und in der Brust absolut und relativ länger sind.

Die Aufstellung von Untertyen ist für die praktische Züchtung nicht erwünscht. Ein Vergleich der Körpermaße von Zuchtkühen der Alpengegenden mit jenen von weiblichen Zuchttieren der Voralpen und des Mittellandes läßt in den meisten Maßen keine wesentlichen Entwicklungsunterschiede erkennen. Allerdings weisen einige Maße mathematisch gesicherte Differenzen auf, welche wahrscheinlich durch den Standort und andere Momente bedingt sind. Der "Alpentypus" besitzt eine größere Brust- und Lendenbreite, sowie einen bedeutenderen Brustumfang, während der "Typus der Voralpen und des Mittellandes" länger in der Brust ist. Sofern allein diese exterieuristischen Körpermerkmale in Betracht gezogen werden, ist es 
verständlich und berechtigt, wenn einzelne Praktiker behaupten, die Herkunft der Tiere mitunter am Exterieur erkennen zu können.

Die Maße von Ausstellungstieren vermögen die durchschnittliche Körperausbildung des Zuchttypus der Rasse nicht wiederzugeben. Sie dürfen daher meines Erachtens für die Standardumschreibung nicht verwendet werden.

Ein Vergleich der Mittelwerte des Vorarlberger Braunviehes (W. Müller) mit meiner Gesamtpopulation beweist, daß die Schweizer Braunviehrasse einen größeren Körperrahmen und ganz besonders eine absolut und relativ bessere Breiten- und Längenentwicklung als die nebengenannte Rasse besitzt. Das Vorarlberger Braunvieh ist relativ kurz, flach und eher hochbeinig, wogegen es in den Tiefenmaßen gut entwickelt ist. Der Körperbau des Vorarlberger Braunviehes weist daranf hin, daß sich dieses auf einer n i edrigeren Z ü chtungsstufe befindet als das Schweizer Braunvieh.

Die Gegenüberstellung der Indexwerte des Zuchttypus des schweizerischen Braunviehes mit jenen aus den Durchschnittsmaßen des Vorarlbergerrindes errechneten gibt eine zahlenmäßige Orientierung bezüglich der Körperproportionen der Ausgangsrasse und dieser Ableger.

In Zukunft sollte nun zunächst der männliche Zuchttypus der Schweizer Braunviehrasse nach ähnlichen Gesichtspunkten, wie es in vorliegender Untersuchung für die weiblichen Rassevertreter geschah, umschrieben werden. Als nächste Etappe in der Analysierung der Schweizer Braunviehrasse ist sodann die Verfolgung des Wachstums der Rasse auf breiter Basis vor und von der Geburt bis zum adulten Stadium, und zwar an denselben Tieren, zu bezeichnen. Soweit mir bekannt ist, hat W. Engler, Stammzuchtbuchführer für Braunvieh, Luzern, bereits entsprechende Untersuchungen begonnen.

Endlich sollte auch die Milchleistung in Menge und Güte, die Mastand Arbeitsleistung, sowie die Gesundheit, Fruchtbarkeit, Zuchtfähigkeit usw. von zuchttypustreuen Tieren der braunen Schweizer Rasse im allgemeinen und nach Herkunft systematisch geordnet untersucht werden. Erst auf Grund dieser Angaben ist eine erschöpfende Rassebeschreibung für das schweizerische Braunvieh denkbar.

Am Schluß dieser Untersuchung möchte ich meinem verehrten Lehrer, Herrn Professor Dr. A. S chmid, E. T. H., Zürich, für die wohlwollende Förderung dieser Arbeit und die vielen wertvollen Ratschläge meinen herzlichen
Dank aussprechen.

Zu besonderem Dank bin ich auch Herrn Professor Dr. M. Küpfer, Vorsteher des landwirtschaftlichen anatomisch-physiologischen Institutes und Herrn Professor Dr. G. Pólya, Dozent für höhere Mathematik an der E. T. H., Zürich verpflichtet.

Endlich danke ich auch der Leitung des Verbandes schweizerischer Braunviehzucht-Genossenschaften in Luzern, der Geschäftsstelle des Verbandes ostschweizerischer Braunviehzucht-Genossenschaften in Wallenstattberg und den Herren J. A nd rea, Direktor der landwirtschaftlichen Schule Plantahof, Landquart, Nationalrat C. Bertschinger, Direktor der Gutswirtschaft Maggi A.-G. in Kemptthal, Dr. W. Eng e ler, Stammzuchtbuchführer für Braunvieh, Luzern, P. A. Knüsel, ing. agr. Pfäffikon, sowie sämtlichen Anstaltsvorstehern und Züchtern, die mir ihre Viehbestände für die vorliegende Arbeit zur Verfügung stellten. 